\title{
Designing a gamified social platform for people living with dementia and their live-in family caregivers
}

\author{
Alexandros T. Tzallas \\ Information Technologies Institute, \\ Centre for Research and Technology \\ Hellas \\ GR55535 \\ Thessaloniki, Greece \\ tzallas@iti.gr \\ Sofia Segkouli \\ Information Technologies Institute, \\ Centre for Research and Technology \\ Hellas \\ GR55535 \\ Thessaloniki, Greece \\ sofia@iti.gr \\ Cristian Barrué \\ Technical University of Catalonia \\ Computer Science Department \\ C/Jordi Girona 1-3 \\ E-08034 \\ Barcelona, Spain \\ cbarrue@cs.upc.edu
}

\author{
Nikolaos Katertsidis \\ Information Technologies Institute, \\ Centre for Research and Technology \\ Hellas \\ GR55535 \\ Thessaloniki, Greece \\ nkaterts@iti.gr
}

Konstantinos Votis

Information Technologies Institute, Centre for Research and Technology

Hellas

GR55535

Thessaloniki, Greece

kvotis@iti.gr

\author{
Konstantinos Glykos \\ Information Technologies Institute, \\ Centre for Research and Technology \\ Hellas \\ GR55535 \\ Thessaloniki, Greece \\ glykos@iti.gr
}

\author{
Dimitrios Tzovaras \\ Information Technologies Institute, \\ Centre for Research and Technology \\ Hellas \\ GR55535 \\ Thessaloniki, Greece \\ tzovaras@iti.gr \\ Ulises Cortés \\ Technical University of Catalonia \\ Computer Science Department \\ C/Jordi Girona 1-3 \\ E-08034 \\ Barcelona, Spain \\ ia@cs.upc.edu
}

\begin{abstract}
In the current paper, a social gamified platform for people living with dementia and their live-in family caregivers, integrating a broader diagnostic approach and interactive interventions is presented. The CAREGIVERSPRO-MMD (C-MMD) platform constitutes a support tool for the patient and the informal caregiver - also referred to as the dyad - that strengthens self-care, and builds community capacity and engagement at the point of care. The platform is implemented to improve social collaboration, adherence to treatment guidelines through gamification, recognition of progress indicators and measures to guide management of patients with dementia, and strategies and tools to improve treatment interventions and medication adherence. Moreover, particular attention was provided on guidelines, considerations and user requirements for the design of a User-Centered Design (UCD) platform. The design of the platform has been based on a deep understanding of users, tasks

This paper is published under the Creative Commons Attribution 4.0 International (CC-BY 4.0) license. Authors reserve their rights to disseminate the work on their personal and corporate Web sites with the appropriate attribution.

PETRA, June 26-29 2018, Corfu

(C) 2018 IW3C2 (International World Wide Web Conference Committee), published under Creative Commons CC BY 4.0 License.

ACM ISBN 978-1-4503-0000-0/18/04

https://doi.org/10.1145/1234567890
\end{abstract}

and contexts in order to improve platform usability, and provide adaptive and intuitive User Interfaces with high accessibility. In this paper, the architecture and services of the C-MMD platform are presented, and specifically the gamification aspects.

\section{CCS CONCEPTS}

- Human-centered computing $\rightarrow$ Human computer interaction (HCI) $\rightarrow$ HCI design and evaluation methods; Laboratory experiments

\section{KEYWORDS}

Dementia, Social Networking, Gamification, Cloud Platform, Caregivers, Interventions; Self-management

\section{ACM Reference format:}

A. T. Tzallas, N. Katertsidis, K. Glykos, S. Segkouli, K. Votis, D. Tzovaras, C. Barrue, U. Cortes. 2018. Designing a gamified social platform for people living with dementia and their live-in family caregivers. In Proceedings of PErvasive Technologies Related to Assistive Environments (PETRA). ACM, New York, NY, USA, 6 pages. 


\section{INTRODUCTION}

Dementia and information and communication technologies (ICT) have apparently not a close similarity, because dementia implies memory or communication difficulties that could make several ICTs not easy to use. Several people living with dementia (PLWD) may also have problems with their ability to see, hear, or even use their hands, and thus experiencing further difficulties on employing ICTs. In addition, PLWD are mostly older, and can be unaccustomed in, or even scared of, using such technologies. Even though all the above issues may be valid, none of them should inhibit the progress and the utilization of ICTs in activities with PLWD. One main task in ICT area for dementia is the efficient communication between PLWD through a wide set of devices available nowadays.

In addition, as dementia affects individuals in different ways [1] a user perspective design becomes challenging in this context. Despite the particular characteristics of PLWD, design landscapes of social platforms for patients with dementia and their caregiver's needs and expectations have not been thoroughly investigated [2]. User interfaces' proper design that can support users' needs while interacting with these systems can enhance social networks' efficiency, as well as users' engagement and navigation to apps [3] . Particularly designing for a specific target group as PLWD constitutes a global challenge. Participation of patients and their caregivers to the design of social platforms' development implies their involvement in the decision -making process of social networks [4]].

In this line. the C-MMD current paper focuses on the design of a social platform based on a user centered approach.

To achieve this all stakeholders and mainly the target groups of patients with dementia and their caregivers have been involved in the design and developmental process in order to implement an intuitive and widely-accepted platform. Moreover, feedback has been collected by patients, carers and doctors in order to customize the platform according to the users' needs.

Furthermore, considering the reduced capabilities of these populations in adapting to the demands of the online ICT platforms like the active assisted living (AAL) service platforms, it is also essential to maximize the adaptability of the related platform architectures [5].

Cloud-based architectures offer easy and constant access to information and services. Nonetheless, not all aspects of PLWD and caregivers' life, and their relations with others can be addressed by a typical cloud computing architecture, consisted of:

- client applications and mobile devices for the front-end,

- $\quad$ servers and storage for the back-end, and

- a clinic intranet and Internet for network.

The platform proposed in this paper provides a unified framework, designed to deliver accessible and highly personalized experiences through a set of intelligent services and applications. CAREGIVERPRO-MMD (C-MDD) is a digital platform, based on social networking, where PLWD, their caregivers, and the medical and social professionals can connect with each other improving the practices of care. In this context, the social platform is the place where user profiles are developed, and social interactions between users are taking place. Thus, the main functionalities of the C-MDD platform include:

- $\quad$ store and manage general and demographic information (account management),

- manage screening results based on questionnaires and scales,

- $\quad$ List medications, and manage medications schedules for treatment adherence.

- Store and manage allergies, medical and social conditions.

- Offer services of a typical social network, as well as gamification elements and services

- Offer a place for therapeutic education and interventions to PLWD and their caregivers.

\section{RELATED WORK}

Other platforms designed to support populations living with impairments, or the elderly and their caregivers follow diverse architecture design approaches. However, they all share some commonalities like the decentralization of the medical and social information, advanced security and privacy and services on demand.

Konstandinidou et al. [] presented a cloud-based architecture to support Active Assisted Living (AAL) services, enhanced by semantic ontologies of the existing scientific knowledge, about people with Mild Cognitive Impairments (MCI) and their needs. The aim is to provide resource sharing under minimal management efforts.

An OSGi-based framework is the heart of the INLIFE project [7], which offers all-around, personalized, multi-faceted ICT solutions and services addressing diverse daily activities (eating, physical activity, commuting, mental stimulation, etc.) to users with cognitive impairment living in their own home or in sheltered homes.

A service-oriented software architecture to supply cloud services to social organizations was also introduced by SOCIALIZE []ㅜ, OASIS [9] , presented a semantically-enabled reference architecture, followed by REMOTE [10] for remote health and social care of elderly living independently.

Perakovic et al. [11] proposed an architecture for user profiling and real-time information management within the AAL concept, in order to offer users a higher level of feeling of safety while on the move.

Apart from architecture requirements targeted to services for people with specific disabilities, others have implemented multinational and context-based services for everyday activities (GoldUI [12]: adaptive embedded human interfaces designed for older people; DOMEO [13]: integrated cloud services for telepresence; SOPRANO [14]: smart devices for elderly and their caregivers). In addition, to overcome cloud isolation, Service Oriented Cloud Computing Architecture (SOCCA) clouds have been introduced that can interoperate with each other [15].

For cognitively impaired people who progressively lose their autonomy, like in C-MMD, a few platforms were structured 
around a dynamic integration of assistive services with their related sensing technologies and interaction devices [16].

Most of the aforementioned examples implement platform architectures for delivering services targeted to specific populations (one user group), or specific needs (e.g. mobility). On the contrary, C-MMD deals with a wide range of user groups, and even wider activities from all aspects of everyday life. In addition, while some AAL platforms introduced architectural components to integrate special hardware equipment, the sensing component of C-MMD is soft (eSurveys, behavioural scales, treatment interventions etc.) and thus, reducing complexity and enhancing extensibility and personalization.

\section{DESIGN METHODOLOGY OF C-MMD}

Various methods have been proposed for the involment of patients with dementia in platform's research and design [17].

In the context of C-MMD, the participatory design method has been adopted as a highly effective method to engage participants, and conceptualize users' expectations and needs [2]

Also, design recommendations as: a. provision for automated support along with explicit information, and $b$. mixed perspective where users can activate the support from the system and confirm this activation, have been employed.

Particularly the mixed iterative user-center design process has been applied [18], including questionnaires and monitoring of $\log$ data to evaluate the usefulness and users' acceptance [19].

Thus, the design and development of the C-MMD platform has taken into account the following features:

- Text size, colour and contrast to make it easy to read even with reduced vision.

- Icons size, colour and underlying meaning, to make it easy to recognize and understand.

- Layout of the GUI distribution, to reduce complexity and make it agile and easy to use.

- Language terminology and sentence structure to make it easy to understand, including the entire localization workout.

- Interaction flows reduced to the minimum to avoid confusion, frustration and complexity.

In terms of functionality adaptation, provision has been made to address:

- The Timing: healthcare professionals provide information about the schedule of appointments and treatment medication; while end-users (patients and caregivers) propose the preferable forms of the reminders.

- The Evaluation Questionnaires: adapt the way the CMMD clinical evaluations works for caregivers to person with mild cognitive impairment, or mild to moderate dementia, in order to obtain quality outcomes for the health professionals, and dedicated content.

However, consideration and evaluation of the end-users' needs late in the design is quite problematic. Usability requirements have to be well and deeply understood in all phases of the design cycle [20]. Hence, the involvement of end-users throughout the design, development and evaluation has been considered as a valuable design feedback for the C-MMD platform.

\section{IMPLEMENTATION OF C-MMD}

CAREGIVERSPRO-MMD (C-MMD) is a UCD social platform, enhanced with gamification elements, for people living with dementia and their live-in family caregivers. Furthermore, the social platform provides diagnostic assessment of dementia as well as interactive interventions.

The aim is to support and develop a mutual assistance community related to dementia across Europe, and especially in France, Italy, Spain and United Kingdom. People use C-MMD to create personal profiles, add other users as "friends", and share information related to dementia with them.

After signing up and adding friends, people communicate with some or all their C-MMD friends by sending private or public messages. Messages can take the form of a "status update" (a post), a private message, a comment about a friend's post or status, or a click of the like button to show support for a friend's update.

Once they learn C-MMD, most users share all kinds of content - photos, videos, and more. They also join C-MMD interest groups related to dementia, in order to communicate with people whom, they might not otherwise know.

Furthermore, users may utilize special C-MMD application to plan events, play games like brain training, and engage in other activities like screening or sharing tailored interventions related to dementia, generate clinical reports for GP, or manage treatments reminders.

The C-MMD user interface (UI) adapts to each user's health conditions and preferences. To provide these functionalities, the C-MMD platform integrates the following services:

- Internationalization and localization.

- Account management.

- Screening.

- Treatment adherence.

- Clinical reporting.

- Social network features.

- Therapeutic education.

- Interactive interventions.

- User interface (UI) adaptation.

Figure 1 depicts the four main layers of the C-MMD platform and their relations.

\subsection{C-MMD Users' Layer}

The C-MMD users' layer includes five categories of stakeholders:

- Admins: IT personnel providing administrative service and platform management.

- Caregivers: people who provide care to PLWD with their activities of daily living. 
- $\quad P L W D$ : people living with dementia, separated into two subgroups with regards to the severity of their symptoms.

- Health Professionals: experts such as doctors, nurses, psychologists, geriatrics, and other specialists, who work as primary care providers of PLWD in hospital, skilled nursing facilities or clinics.

- Social Professionals: experts who work with PLWD and their families to provide care and assistance, and thus improving outcomes in people's lives.

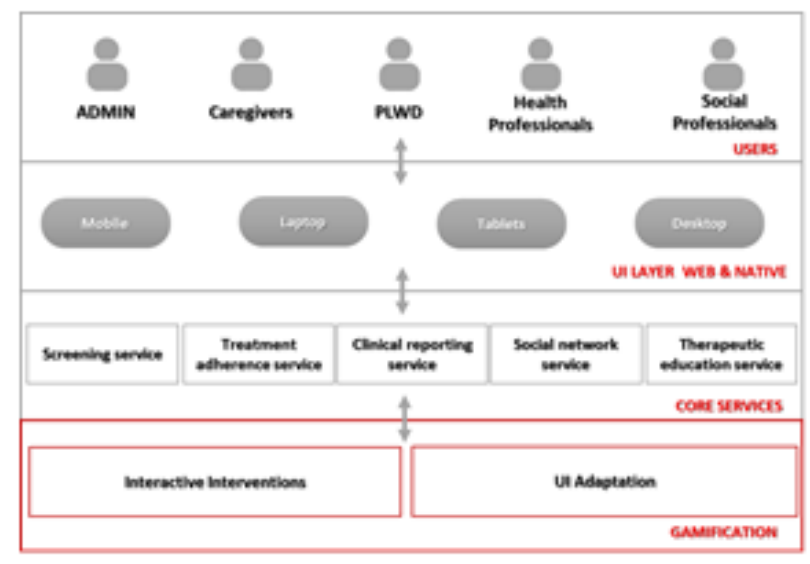

Figure 1: C-MMD Platform Overview

\subsection{C-MMD UI Layer: Web \& Native App}

The user interface (UI) layer consists of all parts that allow the C-MMD users to access, explore and engage with the C-MMD platform's content and services.

The C-MMD platform follows the responsive web design principles and rules. Practically, this involves three main principles that together form the whole responsive design, such as fluid grids, fluid images and media queries. Responsive web design has become more important, as the amount of mobile traffic nowadays accounts for more than half of total Internet traffic.

The C-MMD UI responds to the user's viewport, device or platform. Essentially, it is a design that can attractively acclimate to any screen resolution. A native solution has also been developed for tablets: this presents navigation, content, and functionality in a way specifically optimized for such devices.

\subsection{C-MMD Core Services Layer}

The C-MMD core service delivers the basic outcomes of the platform:

- A screening service for caregivers and people living with memory problems and related symptoms. Memory screening services are essential for anyone concerned about memory loss or experiencing warning signs of dementia; whose family and friends have noticed changes in them; or who believe they are at risk, due to a family history of Alzheimer's disease or a related illness. Screenings are also appropriate for anyone who does not have currently a concern, but would like to determine how his memory is functioning.

- A drug adherence service for drug information management, as well as helping people to improve the adherence to their treatment. This increases PLWD engagement with their condition and medicine, and support them in making effective decisions about their treatment and self-management.

- A tailored intervention service, offering specific interventions based on the user's conditions;

- A clinical reporting service for medical doctors (Figure 2).

- A social network service featuring gamification elements, in order to spread information, ideas, and good practices among C-MMD users.

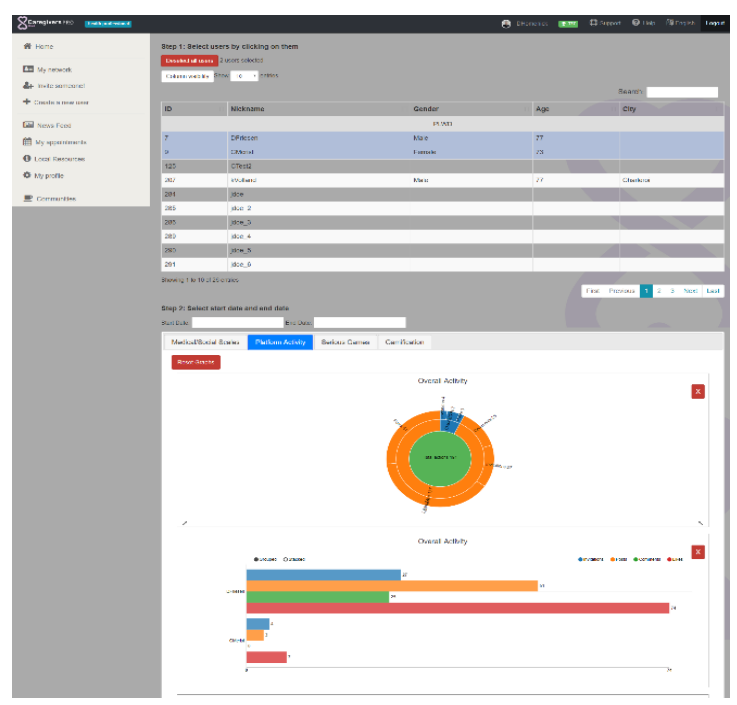

Figure 2: Medical and social professionals can access the reporting service through their user accounts in order to check the current status of the users they take care of (PLWD and Caregivers at risk).

\subsection{C-MMD Gamification Layer}

Gamification is about the application of game elements in non-leisure contexts and for a purpose other than just simple entertainment. For PLWD and people who provide help either as formal caregivers and healthcare professionals or any other form of informal helpers, gamification principles can provide a whole new world to perform and act.

A gamified social environment for people with cognitive decline is an ambitious objective (Figure 3). Thus, the gamification layer consists of two separate components:

- Social games creating motivation for wished user behaviors.

- Serious games providing specific interactive interventions. 


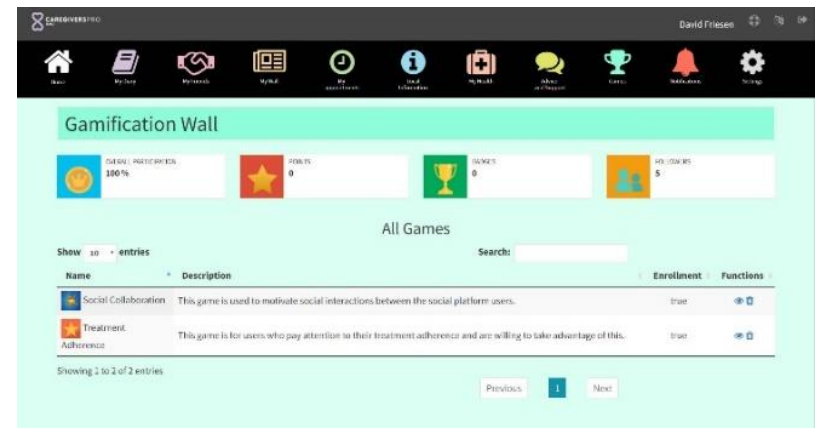

Figure 3: User gamification wall and reward indicators

4.4.1 Social Games: Instead of handling users as separate entities, social games are aware of the "socially constructed presence" that the users of the platform create, and can further enhance their engagement and treatment adherence. Thus, the social gamification service focuses on an incentive scheme based on rewarding models (e.g. points earned for messages posted). A custom engine was implemented for supporting all the games, consisting of the following components:

- The back-end, designed to perform low-level functionality regarding the life cycle of a game, and the gamification interactions of users performed through the social platform.

- The front-end, used to create new gamification proposals and rules by medical and social professionals.

4.4.2 Serious Games: Based on the medical profile of the C-MMD platform users and considering the previous analysis on existent brain fit applications, a set of interactive interventions (serious games) have been developed. These applications are targeted to visuo-spatial short-term memory, orientation, semantic memory, language and problem solving. They are web-based to be easily integrated into the rest of the C-MMD platform, and are operated through either tablets, or personal computers and laptops, similarly to the main C-MMD platform.

\section{A UCD GAMIFICATION DESIGN FRAMEWORK}

A USD centered design is of most importance when designing gamified environments. In fact, the success of gamified platforms depends upon how well users' needs and requirements are well understood. A clear design strategy [21], as well as a careful study of the different motivational cues specific user groups may have for participating on gamified environments [22] is the key to successful engagement of users.

In the game design contexts, Hunicke et al. [23] defined three elements for game design process: (1) Mechanics, (2) Dynamics and (3) Aesthetics.

Cavillo-Gamez in his "Core Elements of the Gaming Experience (CEGE)" [24] considered the user positive experience a core element during the design process.
In addition, most of the gamification frameworks are based on Human-Focused Design principles, positioning the person in the centre of gamification design.

Moreover, gameful interactions as well as social interactions between players are fundamental elements in relation to user interface and experience. However, games can also have a predictive role and be used as clinical assessment tools. Currently serious games have been developed that can assess possible cognitive decline in elderly [25].

In this context, a user-centered gamification framework has been adopted and implemented in C-MMD, as dementia is a multifaceted condition which demands a deep understanding of user profiling.

\section{CONCLUSIONS}

In this paper, the design and architecture of the intelligent and intuitive C-MMD platform were presented, and particularly the enhanced gamification framework and services. Our approach provides an intelligent social gamified platform promoting the quality of life, well-being and medication compliance for people living with memory problems and their caregivers, in the community, at the point of care. The core goal is to establish and develop a mutual assistance community related to dementia across Europe.

\section{ACKNOWLEDGMENTS}

This work has been partially supported by the European Commission through the project HORIZON 2020 - RESEARCH \& INNOVATION ACTIONS (RIA) - 690211 CAREGIVERSPRO-MMD).

\section{REFERENCES}

[1] Kasl-Godley, J. and Gatz, M. Psychosocial interventions for individuals with dementia: an integration of theory, therapy, and a clinical understanding of dementia. Clinical psychology review, 20, 6 (Aug 2000), 755-782.

[2] Aqueasha Martin-Hammond, Foad Hamidi, Tejas Bhalerao, Abdullah Ali, Catherine Hornback, Casey Means, and Amy Hurst. 2017. The Participatory Design of an Adaptive Interface to Support Users with Changing Pointing Ability. In Proceedings of the 19th International ACM SIGACCESS Conference on Computers and Accessibility (ASSETS '17). ACM, New York, NY, USA, 343-344. DOI: https://doi.org/10.1145/3132525.3134810

[3] Hayes, J. R. 2014. User interface design for online social media

[4] Brankaert, R. 2016. Design for dementia: a design-driven living lab approach to involve people with dementia and their context Eindhoven: Technische Universiteit Eindhoven

[5] Barbabella, F., Efthymiou, A., Poli, A., Lancioni, C., Andréasson, F., Salzmann, B., Hanson, E., Döhner, H., Goodwin, F. and Lamura, G. A multilingual web platform supporting informal carers in $27 \mathrm{EU}$ member states. In Proceedings of the 6th AAL Forum 2014 (Bucharest, 2015, 2015). UEFISCDI.

[6] Barrue, C. Cortes, A., Cortes, U. and Tetard, F. Atia Cortés. CAREGIVERSPRO-MMD: Community Services, Helping Patients with Dementia and Caregivers Connect with Others for Evaluation, Support and to Improve the Care Experience. Comp. y Sist. [online]. 2017, vol.21, n.1, pp.23-33. ISSN 1405-5546. http://dx.doi.org/10.13053/cys-21-1-2580

[7] Kaimakamis, E., Karavidopoulou, V., Kilintzis, V., Stefanopoulos, L. and Papageorgiou, V. Development/Testing of a Monitoring System Assisting MCI Patients: The European Project INLIFE. Studies in health technology and informatics, 2422017), 583-586.

[8] SOCIALIZE project. [Retrieved Feb. 2018] Available: http://www.aaleurope.eu/projects/socialize/ 
[9] OASIS project. [Retrieved Feb. 2018] Available: http://www.oasisproject.eu

[10] REMOTE project. [Retrieved Feb. 2018] Available: http://www.aaleurope.eu/projects/remote/

[11] Peraković, D., Periša, M and Bukljaš Skočibušić, M. 2015. Possibilities of implementing Ambient Assisted Living concept in traffic environment // Archives of Transport System Telematics, 8 (2015), 1; 30-34.

[12] GoldUI project. [Retrieved Feb. 2018] Available: http://www.goldui.eu/?lang=en

[13] DOMEO. [Retrieved Feb. 2018] Available: http://www.aaleurope.eu/projects/domeo

[14] SOPRANO project. [Retrieved Feb. 2018] Available: http://cordis.europa.eu/project/rcn/80527_en.html

[15] Tsai, T. C., Lu, J. H., Chen, S. J. and Tang, R. B. Clinical efficacy of house dust mite-specific immunotherapy in asthmatic children. Pediatrics and neonatology, 51, 1 (Feb 2010), 14-18.

[16] Aloulou, H., Mokhtari, M., Tiberghien, T., Biswas, J. and Yap, P. An adaptable and flexible framework for assistive living of cognitively impaired people. IEEE journal of biomedical and health informatics, 18, 1 (Jan 2014), 353-360.

[17] Topo, P.,and Östlund, B. (Eds.). (2009). Dementia, design and technology: time to get involved (Vol. 24). IOS Press.

[18] Shah, S. G. and Robinson, I. User involvement in healthcare technology development and assessment: structured literature review. International journal of health care quality assurance incorporating Leadership in health services, 19, 6-7 2006), 500-515.

[19] De Vito Dabbs, A., Myers, B. A., Mc Curry, K. R., Dunbar-Jacob, J. Hawkins, R. P., Begey, A. and Dew, M. A. User-centered design and interactive health technologies for patients. Computers, informatics, nursing : CIN, 27, 3 (May-Jun 2009), 175-183.

[20] N. Bevan. 1995. Usability is Quality of Use. Advances in Human Factors/Ergonomics, 20, 349-354. doi: 10.1016/S0921-2647(06)80241-8.

[21] Mora, A., Riera, D., Gonzalez, C., \& Arnedo-Moreno, J. (2015, September). A literature review of gamification design frameworks. In Games and virtual worlds for serious applications (VS-Games), 2015 7th international conference on (pp. 1-8). IEEE.

[22] I. Paliokas, A. T. Tzallas, N. Katertsidis, K. Votis, D. Tzovaras. 2017. Gamification in Social Networking: A Platform for People Living with Dementia and their Caregivers. In $17^{\text {th }}$ IEEE International Conference on BioInformatics and BioEngineering, Washington DC, USA, pp. 574-579, doi: 10.1109/BIBE.2017.00104

[23] Hunicke, R., LeBlanc, M. and R. Zubek,.."Mda: A formal approach to game design and game research," AAAI Workshop on Challenges in Game, 2004

[24] Calvillo-Gámez, E. H., Cairns, P., \& Cox, A. L. (2010). Assessing the core elements of the gaming experience. In Evaluating user experience in games (pp. 47-71). Springer London.

[25] Tong, T., Chan, J. H., \& Chignell, M. (2017, April). Serious Games for Dementia. In Proceedings of the 26th International Conference on World Wide Web Companion (pp. 1111-1115). International World Wide Web Conferences Steering Committee. 